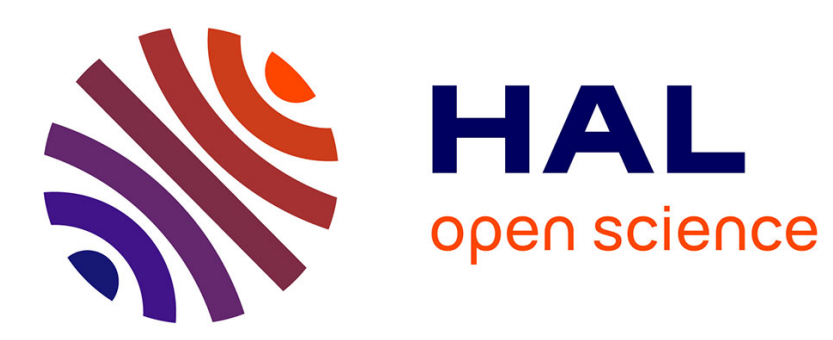

\title{
Randomization vs. selection: How to choose in the absence of preference?
}

Eric Danan

\section{To cite this version:}

Eric Danan. Randomization vs. selection: How to choose in the absence of preference?. Management Science, 2010, 56 (3), pp.503-518. 10.1287/mnsc.1090.1116 . hal-00872249

\section{HAL Id: hal-00872249 \\ https://hal.science/hal-00872249}

Submitted on 11 Oct 2013

HAL is a multi-disciplinary open access archive for the deposit and dissemination of scientific research documents, whether they are published or not. The documents may come from teaching and research institutions in France or abroad, or from public or private research centers.
L'archive ouverte pluridisciplinaire HAL, est destinée au dépôt et à la diffusion de documents scientifiques de niveau recherche, publiés ou non, émanant des établissements d'enseignement et de recherche français ou étrangers, des laboratoires publics ou privés. 


\title{
Randomization versus selection: how to choose in the absence of preference?*
}

\author{
Eric Danan ${ }^{\dagger}$
}

October 13, 2009

\begin{abstract}
Decision-makers sometimes have to choose between alternative options about which they have no preference: either they judge the options equally valuable (indifference) or they have no judgment about their relative value (noncomparability). Choosing randomly is generally considered a natural way to deal with such situations. This paper shows, however, that systematic randomization between noncomparable options may lead to a chain of decisions resulting in monetary losses (a money pump). Furthermore, these losses can be avoided by deliberately selecting one of the noncomparable options instead of randomizing. Thus randomization among noncomparable options is costly relative to deliberate selection. On the other hand, randomization among indifferent options is costless relative to deliberate selection.
\end{abstract}

Keywords. Randomization, selection, money pump, indifference, noncomparability.

JEL Classification. D11.

\section{Introduction}

When a decision-maker (whether an individual, a group, or an organization) has to choose between several options, it is natural that she first try to evaluate these options in order to choose the most valuable one. In complex and changing environments, however, organizations are facing a large number of sophisticated choices and, consequently, managers cannot base all their decisions on an exhaustive evaluation of the various options at stake. How then should a choice be made when there is no clearly identified best option? This paper gives prescriptions for deciding in such situations.

Namely, it will be shown that, when choices have to be made on the basis of a non-exhaustive evaluation of options, some decision strategies, that we will call random, entail potential losses

*Thanks for helpful comments and suggestions to Peter Klibanoff, Jean-François Laslier, Jean-Marc Tallon, Peter Wakker, an associate editor, two referees, as well as seminar audience at the Max Planck Institute for Research into Economic Systems, ESEM 2004, Universitat Pompeu Fabra, Université de Cergy-Pontoise, and Institut Henri Poincarré. Financial support from the French Ministry of Research and the Franco-American Commission for Educational Exchange, as well as hospitality from Northwestern University, Université de Paris 1 Panthéon-Sorbonne, Hong Kong University of Science and Technology, and Universitat Pompeu Fabra, are gratefully acknowledged.

${ }^{\dagger}$ Université de Cergy-Pontoise, THEMA, 33 boulevard du Port, 95000 Cergy-Pontoise, France. E-mail: eric.danan@u-cergy.fr. 
of a specific type, called money pumps, whereas other decision strategies, that we will call selective, protect against these losses. Moreover, this advantage of selective strategies over random strategies is characteristic of non-exhaustive evaluations, since any decision strategy (whether selective or random) based on an exhaustive evaluation of options protects against money pumps. Thus, whereas exhaustiveness of evaluation is sufficient to guide decisions away from money pumps, non-exhaustiveness must be compensated for by a deliberate effort to adopt an appropriate, selective decision strategy.

All this argument, in addition, presupposes that the evaluation of options (whether exhaustive or not) has some form of internal coherence. If the evaluation is incoherent then no decision strategy (neither selective nor random) based on it can protect against money pumps. Thus, the overall prescription in order to avoid money pumps is to (i) start from a coherent evaluation of options and (ii) adopt a selective decision strategy if the evaluation is non-exhaustive.

\subsection{An illustrative example}

For the sake of illustration, we will pursue the following stylized example throughout the paper. A company considers buying a given number of new, identical computers to replace the old, currently installed ones, in order to process certain tasks more efficiently and, thereby, reduce costs. The purchasing department are going to negotiate with the company's usual supplier, on the basis of an evaluation provided by the computing and accounting departments of the decrease in costs expected from different models. Models offered by this supplier only vary according to CPU speed, measured in GigaHertz (GHz), and RAM quantity, measured in GigaBytes (GB), and their CPU speed and RAM quantity are always integers in these units.

Since there are many different combinations of CPU speed and RAM quantity the supplier could potentially offer, the computing and accounting departments have not been able to provide an exhaustive evaluation of all possible models. They have only determined, taking as benchmark the situation in which no computer is bought (i.e. a $0 \mathrm{GHz} \mathrm{CPU}$ and $0 \mathrm{~GB}$ of RAM), that (i) every time CPU speed and RAM quantity are simultaneously increased by 1 $\mathrm{GHz}$ and $1 \mathrm{~GB}$, respectively, costs are reduced by 1 thousand of dollars (k\$) per computer and (ii) increasing $\mathrm{CPU}$ speed alone by $1 \mathrm{GHz}$ or RAM quantity alone by $1 \mathrm{~GB}$ neither increases costs nor reduces them by more than $1 \mathrm{k} \$$.

Note that any model with a CPU speed of $s \mathrm{GHz}$ and a RAM quantity of $q$ GB where $s=q$ is evaluated as reducing costs by precisely $s=q \mathrm{k} \$$ per computer. On the other hand, whenever $s \neq q$, the evaluation only determines that the amount of cost reduction, in $\mathrm{k} \$$ per computer, lies somewhere between the lowest and the highest number among $s$ and $q$. Thus the evaluation is non-exhaustive in the sense that some models are mutually unevaluated: for instance, which of a model with a $1 \mathrm{GHz} \mathrm{CPU}$ and $2 \mathrm{~GB}$ of RAM and another model with a $2 \mathrm{GHz} \mathrm{CPU}$ and 1 GB of RAM is the most valuable (i.e. cost-reducing) is undetermined. Besides, the evaluation is internally coherent in the sense that if a first model is judged more valuable than a second one and the second model is judged more valuable than a third one then, necessarily, the first model is judged more valuable than the third one (this is in fact a higher level of coherence than what is needed to make money pumps avoidable). The purchasing department, in any case, are committed to follow the evaluation. Since the evaluation does not always determine which 
of the available models is the most valuable, they decide that in such cases they will choose a model randomly (say by tossing a coin, more on randomization below).

Negotiation starts and the supplier first makes a take-it-or-leave-it offer for model A, with a $3 \mathrm{GHz}$ CPU and $3 \mathrm{~GB}$ of RAM, for a price of $1.8 \mathrm{k} \$$ per computer. This offer has a net value of $1.2 \mathrm{k} \$$ per computer ( $3 \mathrm{k} \$$ of cost reduction minus $1.8 \mathrm{k} \$$ of price) and, therefore, is naturally accepted by the purchasing department. The supplier then offers the company the possibility to (irreversibly) switch to model $\mathrm{B}$, with $1 \mathrm{GHz}$ and $4 \mathrm{~GB}$, for $0.9 \mathrm{k} \$$ per computer. This new offer has a net value lying between $0.1 \mathrm{k} \$$ and $3.1 \mathrm{k} \$$ (bounds included) per computer (between $1 \mathrm{k} \$$ and $4 \mathrm{k} \$$ of cost reduction minus $0.9 \mathrm{k} \$$ of price) and, hence, it is not clear whether it is more or less valuable than the first offer (i.e. the two models are mutually unevaluated), so the purchasing department choose randomly, and the random choice happens to select the new offer. The supplier then offers to switch from model B to model C, with $2 \mathrm{GHz}$ and $2 \mathrm{~GB}$, for $1 \mathrm{k} \$$ per computer. Again, the third offer, which has a net value of $1 \mathrm{k} \$$ per computer, is not clearly comparable to the second one, so the purchasing department choose randomly, and the random choice happens to select the new offer. The supplier then offers to switch back to model A for $1.9 \mathrm{k} \$$ per computer. This offer has a net value of $1.1 \mathrm{k} \$$ per computer, so it is clearly more valuable than the third offer and, therefore, the purchasing department decide to switch to the new offer. Negotiation stops at this point, so the company ends up buying model A for $1.9 \mathrm{k} \$$ per computer.

Note that, had the company stuck to the first offer by rejecting all subsequent ones, it would also have acquired model A but for only $1.8 \mathrm{k} \$$ per computer. In this sense, the company makes a pure monetary loss of $0.1 \mathrm{k} \$$ per computer. The sequence made of the four successive offers made by the supplier is called a money pump for the company because each offer may be selected over the preceding one according to the company's decision strategy and accepting all successive offers in the sequence is globally impoverishing for the company. Note that the company fell into this money pump without the purchasing department contradicting the provided evaluation, in the sense of choosing a less valuable offer over a more valuable one, at any point during negotiation. Thus, simply following the evaluation does not per se protect the company against money pumps.

Could the purchasing department have avoided this money pump without contradicting the evaluation provided by the computing and accounting departments? The answer is yes, had they been more selective when choosing between mutually unevaluated offers. Indeed, suppose for instance that, instead of choosing randomly between such offers as they systematically did, the purchasing department had decided to assign each offer of $s \mathrm{GHz}$ and $q$ GB for $p \mathrm{k} \$ \mathrm{a}$ (monetary) score equal to $0.5 s+0.5 q-p \mathrm{k} \$$ per computer (note that this score is equal to the value of the offer provided by the computing and accounting departments when $s=q$ and lies within the interval of values provided by them when $s \neq q$ ) and to systematically select the offer with the highest score, only choosing randomly to break ties. Then the second offer above (model B) would have received a score of $1.6 \mathrm{k} \$$, so the purchasing department would have switched from the first to the second offer but not from the second to the third one, thereby ending up buying model B for $0.8 \mathrm{k} \$$ per computer and avoiding the money pump. In fact, the supplier could not possibly construct a sequence of offers that would be a money pump 
against this latter strategy. More generally, whenever choices have to be made on the basis of a non-exhaustive but coherent evaluation, the random decision strategy defined above exposes the company to money pumps whereas some selective strategies are able to protect it against money pumps.

Finally, note that the provided evaluation exhibits ties. For example, a model with $2 \mathrm{GHz}$ and $2 \mathrm{~GB}$ for $1 \mathrm{k} \$$ per computer is just as valuable as a model with $3 \mathrm{GHz}$ and $3 \mathrm{~GB}$ for $2 \mathrm{k} \$$ per computer (both have a net value of $1 \mathrm{k} \$$ per computer). When proposed two such offers, also, the purchasing department could decide either to choose randomly or to deliberately select an offer in some way or another (note that the two decision strategies we have considered both break these ties randomly). These decisions on how to break evaluative ties, however, can neither expose the company to nor protect it against money pumps. In this sense, judging two offers equally valuable or having no judgment about their relative value are two fundamentally different situations.

\subsection{Outline and related literature}

Although we will follow the illustrative example throughout, the analysis is more general: it applies to any choice situation in which a decision-maker first evaluates, exhaustively or not, the various options at stake (evaluation stage) and then decides on her choice behavior based on this evaluation (behavior stage). This two-stage decision process is formalized in Sections 2 and 3. The analysis of money pumps then starts at the behavior stage: Section 4 formally defines money pumps and determines when is a decision-maker's choice behavior invulnerable to money pumps. Moving backwards to the evaluation stage, Sections 5, 6, and 7 determine when does a decision-maker's evaluation make it possible for her choice behavior to be invulnerable to money pumps (without contradicting the evaluation), and when does this possibility depend on the decision-maker adopting a random or selective choice behavior when choosing between mutually unevaluated or equally valuable options. Section 8 concludes with a summary of results and a discussion of managerial implications. Proofs of all results appear in the appendix.

The first money pump argument appears in Davidson, McKinsey, and Suppes (1955). Assuming that the decision-maker has an exhaustive evaluation of options and systematically chooses randomly between equally valuable options, they informally argue that avoiding money pumps requires that the evaluation be transitive. The present analysis formalizes and generalizes their argument by relaxing these two assumptions. In particular, the level of coherence that is needed to avoid money pumps in our framework only requires the evaluation bo be consistent (Suzumura, 1976; Duggan, 1999; Bossert, Sprumont, and Suzumura, 2005), a property equivalent to transitivity when the evaluation is exhaustive but weaker than transitivity when the evaluation is non-exhaustive (see Section 7 for a formal connection with their result). Burros (1974) maintains the exhaustiveness assumption but allows the decision-maker to systematically

refuse to switch from an option to an other, equally valuable one. He finds that avoiding money pumps only requires the evaluation to be acyclic, a property that is weaker than consistency. The importance of choice between equally valuable options in his model, however, comes from a more restrictive definition of money pumps and disappears in the present, more general setup.

Cubitt and Sugden (2001) analyze money pumps in a framework that is more general than 
the present one in several respects, which can be described by means of our illustrative example. In the example, implicitly, we have assumed that the supplier's future offers are not visible to (i.e. foreseen by) the purchasing department. Consequently, they cannot adopt strategies such as "if accepting a new offer implies that merely following the evaluation from then on unavoidably leads us to a money pump, then we will refuse it" (which would lead them to refuse to switch from the second to the third offer). Moreover, since decision strategies consists in determining, once and for all, which of two given offers will be selected (or whether choice will be made randomly), neither can they adopt history-dependent strategies (i.e. strategies in which current choice depends on past choices) such as "if we make a random choice between two offers, then we will keep the randomly selected offer until the supplier makes a strictly more valuable one" (which would also lead them to refuse to switch from the second to the third offer). Cubitt and Sugden (2001) allow for visible and invisible future choices as well as for history-dependent and history-independent strategies. In fact, they show that history-dependence is a powerful way of avoiding money pumps. Our analysis abstracts from such dynamic considerations by presupposing that future choices are invisible and decision strategies are history-independent and, within this restricted framework, investigates to what extent a simple principle, selectivity of choice, can protect against money pumps.

The closest paper to the present one is Mandler (2005). Although with a different terminology and framework, he also shows that a non-exhaustive evaluation of options does not necessarily expose the decision-maker to money pumps. The reason why this is so, however, is different. Namely, the two models have in common that a decision-maker who, based on a non-exhaustive evaluation of options, (i) adopts a history-independent decision strategy and (ii) systematically chooses randomly between mutually unevaluated offers is always vulnerable to money pumps. Mandler (2005) maintains the assumption of systematic randomization between mutually unevaluated offers and shows that adopting a history-dependent behavior can protect the decision-maker against money pumps. Here, on the contrary, we maintain the assumption of history-independent behavior and show that choosing more selectively between mutually unevaluated offers can also protect the decision-maker against money pumps. More recently, Mandler (2009) argues that a decision-maker who breaks evaluative ties randomly is not necessarily exposed to money pumps. Again this is a common conclusion with the present model but coming from different assumptions: Mandler (2009)'s argument relies on history-dependent strategies and only applies to more specific forms of evaluations than those considered here.

The present paper is also closely related to the literature on incomplete preferences, as a non-exhaustive evaluation of offers is in fact modeled by an incomplete preference relation. Aumann (1962) and Bewley (1986) are seminal contributions in this literature, and recent papers include Ok (2002); Dubra and Ok (2002); Dubra, Maccheroni, and Ok (2004); Maccheroni (2004); Kochov (2007); Baucells and Shapley (2008); Manzini and Mariotti (2008); Evren and Ok (2008); Ok, Ortoleva, and Riella (2008); Nascimento and Riella (2008); Nascimento (2008); Chambers (2009). All these models assume transitivity of preference, which is stronger than the consistency property that is necessary to make money pumps avoidable in our framework (for a model of intransitive but consistent preferences, see Manzini and Mariotti, 2004). Concerning choice behavior, it is usual in this literature to assume that any undominated options 
may be chosen, which corresponds to systematic randomization between mutually unevaluated or equally valuable options in our framework. As mentioned above, this assumption has the drawback of being incompatible with invulnerability to money pumps (it has also been experimentally challenged by Ariely, Loewenstein, and Prelec, 2003, 2006). Some models of incomplete preferences allow for history-dependent forms of selection between unevaluated options (Rigotti and Shannon, 2005; Masatlioglu and Ok, 2005; Gursel Tapki, 2007; Lopomo, Rigotti, and Shannon, 2008). Models allowing for history-independent forms of selection between mutually unevaluated options can be found in Gilboa, Maccheroni, Marinacci, and Schmeidler (2008); Evren (2008).

Finally, we might ask how can the company's evaluation and decision strategy, which are taken as primitives in the present analysis, be observed. A possible answer is that, from a prescriptive viewpoint, we can think of them as being simply announced by the company (to a consultant, say). Alternatively, we can stand in line with the classical revealed preference approach and derive them from observable choice behavior. The decision strategy, then, corresponds to the usual concept of revealed preference directly modeling behavior in binary choice situations, as derived from a choice function (Richter, 1971; Sen, 1971), provided that randomization is observable as well. The evaluation, on the other hand, is not directly observable and, hence, must be derived indirectly from behavior. Eliaz and Ok (2006) propose a method for doing so, but this method crucially relies on the assumption of systematic randomization between mutually unevaluated or equally valuable options and, hence, is incompatible with the present analysis. A method allowing for deliberate selection between mutually unevaluated or equally valuable options can be found in Danan (2003a,b, 2008).

\section{Setup}

In this section we introduce the two formal ingredients of the analysis to come: options and preference relations. Options are the objects among which the decision-maker has to choose, and preference relations over these options will be used to model both the evaluation stage and the behavior stage of the decision process.

\subsection{Options}

The decision-maker faces some set $X$ of options. More specifically, we have $X=B O \times M P$, where $B O$ is some set of basic objects and $M P$ is some set of monetary payoffs, so that an option $x=(b, m) \in X$ specifies both a basic object $b \in B O$ and a monetary payoff $m \in M P$ to be received by the decision maker. The set $B O$ of basic objects is totally arbitrary (so it may be finite or infinite, countable or uncountable...) and the set $M P$ of monetary payoff can be any open set of real numbers (such as the set of all real numbers, for instance). Options will be denoted by $x, y \ldots$, basic objects by $b, c \ldots$, and monetary payoffs by $m, n \ldots$

In our illustrative example, the decision-maker is the company and the options are the supplier's offers. An offer $x=(b, m) \in X$ specifies both a computer model $b \in B O$ and a (negative) monetary payoff $m \in M P$ to be received by the company (i.e. the price per computer paid to the supplier). Thus we can take $M P$ to be the set of all negative real numbers (we 
could as well take a smaller set if, for example, there is an a priori upper bound on the price). Concerning basic objects, since computers only differ by CPU speed and RAM quantity, we can take $B O$ to be the set of all pairs of nonnegative integers (we could similarly impose upper bounds, or not restrict attention to integers...), so that a model $b$ with a $s \mathrm{GHz}$ CPU and $q \mathrm{~GB}$ or RAM is denoted by $b=(s, q)$. Then an offer for model $b=(s, q)$ at price $p \mathrm{k} \$$ per computer is denoted by $x=((s, q),-p)$ or, letting $m=-p \in M P$, by $x=((s, q), m)$.

From now on, we will use the specific terminology of the illustrative example (company, offers...) rather than the general one (decision-maker, options...) throughout the text. The reader should keep in mind, however, that the analysis is of course more general than the example.

\subsection{Preference relations}

Preferences over $X$ (whether evaluative or behavioral, see below) are modeled by means of a binary relation on $X$ generically denoted by $R$, i.e. given two offers $x$ and $y$, we write $x R y$ if offer $x$ is weakly preferred to offer $y$.

Definition. The strict preference relation $P(R)$, indifference relation $I(R)$, and noncomparability relation $N(R)$ of $R$ are defined by, for any $x, y \in X$ :

- $x P(R) y$ if and only if $x R y$ and not $y R x$,

- $x I(R) y$ if and only if $x R y$ and $y R x$,

- $x N(R) y$ if and only if neither $x R$ y nor $y R x$.

Thus given two offers $x$ and $y$, one and only one of the following situations must arise: either $x$ is strictly preferred to $y(x P(R) y)$, or $y$ is strictly preferred to $x(y P(R) x)$, or $x$ and $y$ are indifferent $(x I(R) y)$, or $x$ and $y$ are noncomparable $(x N(R) y)$. We now introduce two groups of properties that a preference relation may or may not satisfy. Some of these properties will be assumed throughout the analysis, whereas others will turn out to be related to the possibility for the company to avoid money pumps. Properties in the first group are general in the sense that they do not depend on the specific structure of the set $X$ of offers (i.e. they can be stated for any set $X$ of options, without assuming $X=B O \times M P$ ). Properties in the second group are monetary in the sense that they explicitly rely on the specific structure of offers and, in particular, on their monetary component.

\subsubsection{General properties of preference relations}

We denote by $T(R)$ the transitive closure of $R$, i.e. we write $x T(R)$ y if either offer $x$ is weakly preferred to offer $y$, or offer $x$ is weakly preferred to some offer that is weakly preferred to offer $y$, and so on for any finite sequence of offers:

Definition. The transitive closure relation $T(R)$ of $R$ is defined by, for any $x, y \in X$, $x T(R) y$ if and only if there exist some integer $k \geq 1$ and some sequence $x_{0}, x_{1}, \ldots, x_{k} \in X$ such that $x_{0} R x_{1}, \ldots, x_{k-1} R x_{k}, x_{0}=x$, and $x_{k}=y$. 
Note that this definition can be applied to any binary relation on $X$, so we can, for example, define the transitive closure $T(P(R))$ of the strict preference relation $P(R)$ of $R$. Note also that $x R y$ implies $x T(R) y$ by definition (taking $k=1$ ), but the reverse implication (which is the transitivity property defined below) is not necessarily true.

Definition. $R$ is:

- reflexive if, for any $x \in X, x I(R) x$,

- complete if there exist no $x, y \in X$ such that $x N(R) y$,

- acyclic if, for any $x, y \in X$, if $x T(P(R))$ y then not $y P(R) x$,

- consistent if, for any $x, y \in X$, if $x T(R)$ y then not $y P(R) x$,

- transitive if, for any $x, y \in X$, if $x T(R)$ y then $x R y$.

Clearly, completeness implies reflexivity (since $x P(R) x$ is impossible by definition of $P(R)$, an equivalent definition of reflexivity is that $x N(R) x$ for no $x \in X)$. Also, transitivity implies consistency and consistency implies acyclicity. Finally, transitivity and consistency are equivalent if $R$ is complete. To illustrate the differences between the last three properties, simply take $X=\{x, y, z\}$ (forgetting for a moment our assumption that $X=B O \times M P$ ) and consider a preference relations $R$ on $X$ such that $x P(R) y$ and $y P(R) z$. If $z P(R) x$ then $R$ is cyclic. If $z I(R) x$ then $R$ is acyclic but inconsistent. If $z N(R) x$ then $R$ is consistent but intransitive. Finally, if $x P(R) z$ then $R$ is transitive.

\subsubsection{Monetary properties of preference relations}

We denote by $M(R)$ the monetary closure of $R$, i.e. we write $(b, m) M(R)(c, n)$ if any offer for model $b$ with a monetary payoff strictly higher than $m$ (i.e. at a price strictly lower than $-m$ $\mathrm{k} \$$ per computer) is strictly preferred to an offer for model $c$ with monetary payoff $n$ :

Definition. The monetary closure relation $M(R)$ of $R$ is defined by, for any $(b, m),(c, n) \in$ $X,(b, m) M(R)(c, n)$ if and only if, for any $o \in M P$ such that $o>m,(b, o) P(R)(c, n)$.

Note that $(b, m) R(c, n)$ does not necessarily imply $(b, m) M(R)(c, n)$ (this implication is the monotonicity property defined below), and neither is the reverse implication (which is a continuity property not needed in this paper) necessarily true.

Definition. $R$ is:

- monotonic if, for any $(b, m),(c, n) \in X$, if $(b, m) R(c, n)$ then $(b, m) M(R)(c, n)$,

- Archimedean if, for any $(b, m),(c, n) \in X$ such that $(b, m) R(c, n)$ and any $o \in M P$ such that $o>m$, there exists some $p \in M P$ such that $p>n$ and $(b, o) R(c, p)$.

Monotonicity means, roughly speaking, that more money is always strictly preferred to less money: if offer $(b, m)$ is weakly preferred to offer $(c, n)$ and offer $(b, m)$ 's monetary payoff is increased from $m$ to any $o>m$ (our assumption that $M P$ is an open set guarantees that we 
can always increase the monetary payoff), then offer $(b, o)$ is strictly preferred to offer $(c, n)$. Archimedeanness adds that we can in fact increase offer $(c, n)$ 's monetary payoff from $n$ to some $p>n$ while still having offer $(b, o)$ weakly preferred to offer $(c, p)$. The intuition is that if we take $p$ close enough to $n$ (our assumption that $M P$ is open guarantees that we can take $p$ as close to $n$ as we want), then the effect of the increase from $n$ to $p$ will be small enough so as not to overbalance the effect of the increase from $m$ to $o$. Thus Archimedean here is in the sense that small changes in monetary payoff have a small effect on preference.

\section{The two-stage decision process}

The company follows a two-step decision process: first, it (the computing and accounting departments) comes up with judgments of the form "I judge offer $x$ more valuable than offer $y "$ (evaluation stage) and, second, based on these judgments, it (the purchasing department) makes decisions of the form "I will choose offer $x$ if I have to choose between offer $x$ and offer $y$ " (behavior stage). Each of these two stages will be modeled by means of a preference relation, and the fact that choice behavior is based on evaluation will be reflected by some relationships between these two preference relations.

\subsection{Evaluation stage}

We model the evaluation stage by means of an evaluative preference relation $E$ on $X$, i.e. we write $x E y$ if the computing and accounting departments judge offer $x$ at least as valuable as offer $y$. $x P(E) y$ then means that they judge offer $x$ strictly more valuable than offer $y$, $x I(E) y$ that they judge offer $x$ and offer $y$ equally valuable, and $x N(E) y$ that they have not mutually evaluated offer $x$ and offer $y$.

In the illustrative example, the evaluation provided by the computing and accounting departments corresponds to the evaluative preference relation $E_{1}$ defined by $((s, q), m) E_{1}((t, r), n)$ if and only if either $s+m=t+n$ and $q+m=r+n$, or $s+m>t+n$ and $q+m>r+n$. This evaluative preference relation is incomplete, reflecting non-exhaustiveness of the evaluation: for example, the first and second offers made by the supplier are noncomparable, i.e. $((3,3),-1.8) N\left(E_{1}\right)((1,4),-0.9)$ (indeed, we have $3-1.8=1.2>1-0.9=0.1$ and $3-1.8=1.2<4-0.9=3.1)$. Note that we also count as noncomparability the case in which $s+m \geq t+n$ and $q+m \geq r+n$ with one equality and one strict inequality, as in this case it is undetermined whether the first offer is equally or more valuable than the second one (except in Section 7 , we could as well count this as strict preference by defining $((s, q), m) E_{1}((t, r), n)$ if and only if $s+m \geq t+n$ and $q+m \geq r+n)$. One can also check that this evaluative preference relation is reflexive, transitive, monotonic, and Archimedean.

We will assume throughout the analysis that the company's evaluative preference relation $E$ is reflexive and (except in Section 7) monotonic and Archimedean. Reflexivity merely means that any offer is judged equally valuable to itself. Monotonicity and Archimdeanness are also natural assumptions in this context: they simply mean that money is positively valued, and that a small change in an offer's price has a small effect on its value. 


\subsection{Behavior stage}

Similarly, we model the behavior stage by means of a behavioral preference relation $B$ on $X$, i.e. we write $x B y$ if the purchasing department either decide that they will select offer $x$ or that they will choose randomly if they have to choose between offer $x$ and offer $y \cdot x P(B) y$ then means that they decide to select offer $x, x I(B) y$ that they decide to choose randomly, and $x N(B) y$ that they decide neither to select an offer nor to choose randomly (we will in fact rule out this last possibility by assumption).

The simplest way of interpreting randomization is that the purchasing department will, each time they have to choose between two behaviorally indifferent offers, select one of these two offers by tossing a coin. The coin may be fair or unfair (as long as both sides have positive probability), the company may use always the same coin or a different one each time, but it is essential that there be a separate, independent coin toss each time (so that if $x I(B) y$ and $z I(B) a$ and we already know that the coin toss between $x$ and $y$ has selected $x$, we still do not know whether the coin toss between $z$ and $a$ will select $z$ or $a$ ). We can also consider other ways of randomizing, such as delegating each choice to a different person (as long as their choice behaviors are uncorrelated), or choosing on the spur of the moment (to the extent that this makes choices independent of each other).

In the illustrative example, the second, selective decision strategy the purchasing department could have adopted corresponds to the behavioral preference relation $B_{2}$ defined by $((s, q), m) B_{2}((t, r), n)$ if and only if $0.5 s+0.5 q+m \geq 0.5 t+0.5 r+n$. One can check that this behavioral preference relation is complete, transitive, monotonic, and Archimedean. The first, random decision strategy that they adopted instead, on the other hand, corresponds to the behavioral preference relation $B_{1}$ defined by $((s, q), m) B_{1}((t, r), n)$ if and only if not $((t, r), n) P\left(E_{1}\right)((s, q), m)$, i.e. if and only if either $s+m \geq t+n$ or $q+m \geq r+n$. This behavioral preference relation is complete and Archimedean, but neither transitive nor monotonic.

We will assume throughout the analysis that the company's behavioral preference relation $B$ is complete and (except in Section 7) Archimedean. Completeness simply means that choice situations are binding: if the purchasing department have to choose between two offers then, unavoidably, they must either select one offer or choose randomly. Archimedeanness, on the other hand, is perhaps less compelling here than at the evaluative stage, as the rationale for Archimedeanness is naturally linked to the value of money but choice behavior does not necessarily reflect an underlying evaluation. For example, in the extreme case in which there is no evaluation at all, there seems to be no obvious reason why any particular pattern of behavior (possibly violating Archimedeanness) should not occur. Yet there are many situations in which behavioral preferences will naturally satisfy the Archimedeanness assumption, such as whenever they are determined by some monetary score of the form $f(b)+m$ where $f$ is any function assigning to each model a monetary score independently of the offer's monetary payoff (which is the case in the illustrative example). Thus we can see Archimedeanness here as a restriction to sufficiently well-behaved choice behavior, restriction that is in any case necessary to obtain the results to come. 


\subsection{From evaluation to behavior}

If the computing and accounting departments have judged offer $x$ strictly more valuable than offer $y$ and the purchasing department have to choose between $x$ and $y$, then it is sensible that they do not contradict this judgment and, therefore, select offer $x$. We will assume that $B$ obeys $E$ in this sense:

Definition. $B$ obeys $E$ if, for any $x, y \in X$, if $x P(E)$ y then $x P(B) y$.

On the other hand, if the computing and accounting departments have not mutually evaluated offers $x$ and $y$, then no constraint is imposed on the purchasing department's choice behavior: they may either choose randomly between $x$ and $y$, or deliberately select $x$, or deliberately select $y$. We will say that the they resolve noncomparability randomly in the first case and that they resolve noncomparability selectively in the last two cases. As a special case, it is possible that the purchasing department systematically resolve noncomparability randomly rather than selectively:

Definition. $B$ resolves all noncomparabilities randomly if, for any $x, y \in X$, if $x N(E) y$ then $x I(B) y$.

Similarly, if the computing and accounting departments have judged offer $x$ and offer $y$ equally valuable, then the purchasing department may either resolve indifference randomly or resolve indifference selectively. As a special case, it is possible that they systematically resolve indifference randomly rather than selectively:

Definition. $B$ resolves all indifferences randomly if, for any $x, y \in X$, if $x I(E) y$ then $x I(B) y$.

Figure 1 illustrates the relationship between the company's evaluation and behavior for two given, distinct offers $x$ and $y$. The four ovals correspond to all possible evaluative judgments concerning $x$ and $y$. The three rectangles correspond to all possible choice behaviors concerning $x$ and $y$. From a given evaluative judgment, an arrow points to each choice behavior obeying it. There is only one possibility in case of evaluative strict preference (behavior is fully determined by evaluation), and three possibilities in case of evaluative noncomparability or indifference (behavior is not constrained at all by evaluation). The two dashed arrows correspond to selective resolutions of noncomparability and the two dotted arrows to selective resolutions of indifference. Note that if noncomparability and indifference are both resolved randomly, then behavior is fully determined by evaluation.

In the illustrative example, it is easy to see that the two behavioral preference relations $B_{1}$ and $B_{2}$ both obey the evaluative preference relation $E_{1} . B_{1}$, in addition, resolves all noncomparabilities and all indifferences randomly and, accordingly, is fully determined by $E_{1}$. As mentioned in the introduction, $B_{2}$ also resolves all indifferences randomly, but resolves some noncomparabilities selectively (hence it is not fully determined by $E_{1}$ ). Note that it does also resolve some noncomparabilities randomly (between offers $((1,2),-1)$ and $((2,1),-1)$, for instance). Globally, $B_{2}$ is more selective than $B_{1}$. 


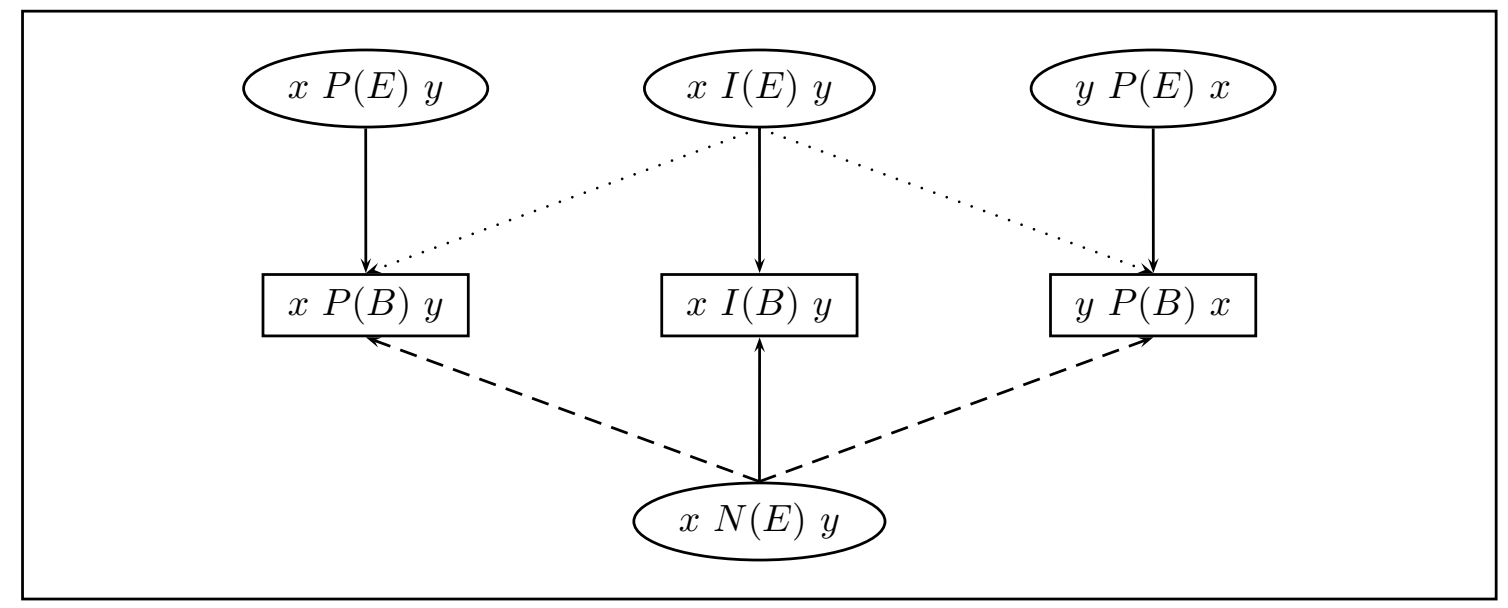

Figure 1: From evaluation to behavior.

\section{Money pumps and choice behavior}

In this section we formally define money pumps and determine when is the company's behavioral preference relation $B$ vulnerable or invulnerable to money pumps. This will enable us to subsequently determine when, and how, does the company's evaluative preference relation $E$ make it possible for it to adopt a behavioral preference relation that obeys $E$ and is invulnerable to money pumps.

If the purchasing department hold an offer $(b, m)$ and are proposed by the supplier to exchange it for a new offer $(c, n)$, then they may accept the exchange if and only if $(c, n) B(b, m)$ (i.e. either $(c, n) P(B)(b, m)$ and they select the new offer or $(c, n) I(B)(b, m)$ and they choose randomly between the two offers, possibly ending up with the new one). A money pump for the company is a sequence of exchanges that are individually acceptable but globally impoverishing, i.e. a sequence of the form $(b, n) B(b, m)$ with $n<m$, or $(b, o) B(c, n) B(b, m)$ with $o<m$, and so on for any finite sequence of offers. We will say that $B$ is invulnerable to money pumps if no such sequence can be constructed:

Definition. $B$ is invulnerable to money pumps if there exist no $b \in B O$ and $m, n \in M P$ such that $n<m$ and $(b, n) T(B)(b, m)$.

The following proposition gives a necessary and sufficient condition for $B$ to be invulnerable to money pumps:

Proposition 1. Assume that $B$ is complete and Archimedean. Then $B$ is invulnerable to money pumps if and only if $B$ is monotonic and $M(B)$ is transitive.

Proposition 1 provides a simple way of checking whether the purchasing department's decision strategy is vulnerable to money pumps or not. In the illustrative example, the behavioral preference relation $B_{1}$ is, as noted above, complete and Archimedean but not monotonic, so it is vulnerable to money pumps (see the introduction). In fact, one can check that neither is $M\left(B_{1}\right)$ transitive, which is another reason why $B_{1}$ is vulnerable to money pumps. The behavioral preference relation $B_{2}$, on the other hand, is complete, Archimedean, monotonic, and 
transitive. Moreover, one can check that $M\left(B_{2}\right)=B_{2}$, so $M\left(B_{2}\right)$ is obviously transitive and, hence, $B_{2}$ is invulnerable to money pumps by Proposition 1 . In general, a behavioral preference relation is not necessarily equal to its monetary closure, but it turns out that completeness, Archimedeanness, monotonicity, and transitivity of the behavioral preference relation together imply transitivity of its monetary closure and, consequently, guarantee invulnerability to money pumps:

Proposition 2. Assume that $B$ is complete, Archimedean, and monotonic. If $B$ is transitive, then $B$ is invulnerable to money pumps.

The converse of Proposition 2 is not true: a complete, Archimedean, and monotonic behavioral preference relation can be invulnerable to money pumps (i.e. have a transitive monetary closure) without being transitive. This can be seen by simply amending the behavioral preference relation $B_{2}$ of the illustrative example as follows: suppose the purchasing department decide that if they have to choose between two offers that have the same monetary score they will, instead of systematically choosing randomly, break the tie more selectively by (i) computing the difference in CPU speed between the two models and (ii) choosing the model with the fastest CPU if this difference is odd and the model with the slowest CPU if this difference is even (if both computers have the same CPU speed they will still choose randomly). Then one can check that the behavioral preference relation $B_{3}$ corresponding to this amended strategy is complete, Archimedean, monotonic, and that $M\left(B_{3}\right)=M\left(B_{2}\right)$, so $B_{3}$ is invulnerable to money pumps. Yet $B_{3}$ is intransitive: for instance, we have $((3,1),-1) B_{3}((2,2),-1)$ and $((2,2),-1) B_{3}((1,3),-1)$ but $((1,3),-1) P\left(B_{3}\right)((3,1),-1)$.

Thus, whether a complete, Archimedean, and monotonic behavioral preference relation $B$ is invulnerable to money pumps or not is primarily a property of its monetary closure $M(B)$. In other words, different such behavioral preference relations having the same monetary closure (as in the example just above) are either all vulnerable or all invulnerable to money pumps (this is an immediate consequence of Proposition 1). In fact, as far as invulnerability to money pumps is concerned, the purchasing department could equivalently use $M(B)$ rather than $B$ as decision strategy:

Proposition 3. Assume that $B$ is complete, Archimedean, and monotonic. Then $B$ is invulnerable to money pumps if and only if $M(B)$ is invulnerable to money pumps.

\section{Money pumps and evaluative judgments}

We now turn to the evaluation stage of the company's decision process. Because the purchasing department's decision strategy is not yet determined at this stage, we cannot, strictly speaking, determine whether the company is exposed to money pumps or not. Nevertheless, if the evaluation provided by the computing and accounting departments is such that any decision strategy based on it is necessarily vulnerable to money pumps, then we can conclude that the evaluation per se exposes the company to money pumps. Thus we first investigate whether the company's evaluative preference relation $E$ is potentially invulnerable to money pumps in this sense: 
Definition. $E$ is potentially invulnerable to money pumps if there exists some complete and Archimedean $B$ that is invulnerable to money pumps and obeys $E$.

The following proposition gives a sufficient condition for $E$ to be potentially invulnerable to money pumps:

Proposition 4. Assume that $E$ is reflexive, monotonic, and Archimedean. If $M(E)$ is consistent, then $E$ is potentially invulnerable to money pumps.

In the illustrative example, the evaluative preference relation $E_{1}$ is, as noted above, reflexive, monotonic, Archimedean, and transitive. Moreover, one can check that $M\left(E_{1}\right)$ is transitive as well. Since transitivity implies consistency, it follows that $E_{1}$ is potentially invulnerable to money pumps, so the purchasing department can possibly adopt a decision strategy based on $E_{1}$ that is invulnerable to money pumps (in fact, the behavioral preference relation $B_{2}$ corresponds to such a decision strategy). This does not imply, however, that any decision strategy based on $E_{1}$ is necessarily invulnerable to money pumps (in fact, the behavioral preference relation $B_{1}$ corresponds to a decision strategy also based on $E_{1}$ but that is vulnerable to money pumps). Thus whether the company will actually be exposed to money pumps or not depends on which decision strategy the purchasing department will actually adopt.

As at the behavior stage, an evaluative preference relation is not, in general, equal to its monetary closure (in fact we have $M\left(E_{1}\right) \neq E_{1}$ in the illustrative example). Now, in addition, transitivity of a reflexive, monotonic, and Archimedean evaluative preference relation neither implies nor is implied by transitivity of its monetary closure. Still, transitivity of $E$ turns out to be also sufficient for $E$ to be potentially invulnerable to money pumps:

Proposition 5. Assume that $E$ is reflexive, monotonic, and Archimedean. If $E$ is consistent, then $E$ is potentially invulnerable to money pumps.

Consistency of $E$ expresses a weak form of internal coherence of the evaluation: roughly speaking, if offer $x$ is judged at least as valuable as offer $y$ and offer $y$ is judged at least as valuable as offer $z$ then offer $z$ must not be judged strictly more valuable than offer $x$. Violations of this property, it seems, would presumably result from errors in the evaluation process. In fact, there are many situations in which the evaluative preference relation will naturally even be transitive, such as whenever offers are evaluated through a given set of criteria, each offer receiving a numerical score in each criterion, and offer $x$ is judged more valuable than offer $y$ if and only if $x$ has a higher score than $y$ in each criterion (which is the case in the illustrative example). Situations where the evaluative preference relation are intransitive but consistent are also conceivable (Manzini and Mariotti, 2004).

The converses of Propositions 4 and 5 are not true: a reflexive, monotonic, and Archimedean evaluative preference relation can be potentially invulnerable to money pumps even without being consistent and without having a consistent monetary closure. In the illustrative example, this would be the case if the computing and accounting departments evaluated offers as follows: (i) offer $((s, q), m)$ is at least as valuable as offer $((t, r), n)$ if and only if both $m \geq n$ and model $(s, q)$ is at least as valuable as model $(t, r)$ and (ii) between two models, the most valuable one is the one with the fastest CPU if the difference in CPU speed between the two models is odd, and 
the one with the slowest CPU if this difference is even (if the two models have the same CPU speed then they are equally valuable). One can check that the evaluative preference relation $E_{2}$ corresponding to this evaluation is reflexive, monotonic, and Archimedean, but not consistent (in fact, it is even cyclic), and that $M\left(E_{2}\right)=E_{2}$, so $M\left(E_{2}\right)$ is not consistent either. Yet $E_{2}$ is potentially invulnerable to money pumps. Indeed, suppose the purchasing department adopted the following decision strategy: among two offers, (i) always select the offer with the highest monetary payoff, if there is one, and (ii) if both offers have the same monetary payoff, select the one with the most valuable model (choosing randomly if the two models are equally valuable). Then the behavioral preference relation $B_{4}$ corresponding to this decision strategy is complete, Archimedean, invulnerable to money pumps, and obeys $E_{2}$.

As at the behavior stage, whether a reflexive, monotonic, and Archimedean evaluative preference relation $E$ is potentially invulnerable to money pumps or not is primarily a property of its monetary closure $M(E)$ and, as far as potential invulnerability to money pumps is concerned, the computing and accounting departments could equivalently use $M(E)$ rather than $E$ as evaluation:

Proposition 6. Assume that $E$ is reflexive, monotonic, and Archimedean. Then $E$ is potentially invulnerable to money pumps if and only if $M(E)$ is potentially invulnerable to money pumps.

In any case, non-exhaustiveness of evaluation does not preclude invulnerability to money pumps and, in this sense, is not costly per se. However, as we will see in the next section, nonexhaustiveness of evaluation calls for specific choice behavior in order to avoid money pumps.

\section{Money pumps and selectivity of choice}

If the computing and accounting departments' evaluation is potentially invulnerable to money pumps then some, but not necessarily all decision strategies based on it are able to protect the company from money pumps. Moreover, as mentioned above, different decision strategies based on the same evaluation can only differ in the way they choose between mutually unevaluated or equally valuable offers. Hence whether the company will actually be exposed to money pumps or not entirely depends on the (random or selective) way the purchasing department will resolve noncomparabilities and indifferences. In this section we investigate how resolution of noncomparability and indifference affects invulnerability to money pumps.

Among all decision strategies based on a given evaluation, the simplest is, arguably, the one that resolves all noncomparabilities and all indifferences randomly (in the illustrative example, this corresponds to the behavioral preference relation $B_{1}$ ). Indeed, this strategy can be simply implemented as follows: among two offers, select the most valuable if there is one, and toss a coin otherwise. Any other decision strategy based on the evaluation, on the other hand, requires a deliberate effort to resolve some noncomparabilities and/or indifferences selectively. But at the same time, resolving some noncomparability or indifference selectively rather than randomly implies accepting less exchanges (if the purchasing department decide to choose randomly between offers $x$ and $y$ they may both accept to exchange $x$ for $y$ and $y$ for $x$, whereas if they decide to deliberately select one of these two offers they will only accept to exchange the 
unselected offer for the selected one but not the other way around). Since money pumps are sequences of acceptable exchanges, this can only reduce the company's vulnerability to money pumps or leave it unchanged but not increase it.

Thus the question under investigation becomes: how selective must the purchasing department be in order to protect the company from money pumps? In particular, is it possible to resolve all noncomparabilities and/or all indifferences randomly without being exposed to money pumps?

Definition. E is potentially invulnerable to money pumps without selective resolution of noncomparability (resp. indifference) if there exists some complete and Archimedean $B$ that is invulnerable to money pumps, obeys $E$, and resolves all noncomparabilities (resp. indifferences) randomly.

The following proposition gives a necessary condition for money pumps to be avoidable while resolving all noncomparabilities randomly:

Proposition 7. Assume that $E$ is reflexive, monotonic, and Archimedean. If $E$ is potentially invulnerable to money pumps without selective resolution of noncomparability, then $M(E)$ is complete and transitive.

In the illustrative example, the evaluative preference relation $E_{1}$ is reflexive, monotonic, Archimedean, and such that $M\left(E_{1}\right)$ is transitive but incomplete, so it is potentially invulnerable to money pumps but not without selective resolution of noncomparability. Hence the behavioral preference relation $B_{1}$, which resolves all noncomparabilities randomly, is necessarily vulnerable to money pumps. To avoid money pumps, the company must adopt a behavioral preference relation that resolves at least some noncomparabilities selectively, such as $B_{2}$.

Completeness of $M(E)$ implies that $E$ is almost complete (i.e. the evaluation is almost exhaustive), in the sense that if two offers are noncomparable then, for at least one of the two offers, any (however small) change in monetary payoff makes it comparable to the other offer. Transitivity of $M(E)$, on the other hand, is just equivalent to its consistency since $M(E)$ is complete. As strong as these two properties are, the converse of Proposition 7 is not true: an example can be constructed showing that a behavioral preference relation $E$ that is reflexive, monotonic, Archimedean, and such that $M(E)$ is complete and transitive is not necessarily potentially invulnerable to money pumps without selective resolution of noncomparability. Comparing Propositions 4 and 7, it appears that whenever the monetary closure of the evaluative preference relation is consistent but not complete, avoiding money pumps is possible but requires selective resolution of at least some noncomparabilities:

Proposition 8. Assume that $E$ is reflexive, monotonic, and Archimedean. If $M(E)$ is consistent but not complete, then $E$ is potentially invulnerable to money pumps but not without selective resolution of noncomparability.

The following proposition shows that, unlike resolution of noncomparability, resolution of indifference does not affect invulnerability to money pumps in any way: 
Proposition 9. Assume that $E$ is reflexive, monotonic, and Archimedean. If $E$ is potentially invulnerable to money pumps, then $E$ is potentially invulnerable to money pumps without selective resolution of indifference.

In the illustrative example, the behavioral preference relation $B_{3}$ obeys the evaluative preference relation $E_{1}$ and resolves some indifferences selectively. Since it is invulnerable to money pumps, there must exist, by Proposition 9, another behavioral preference relation that is invulnerable to money pumps, obeys $E_{1}$, and resolves all indifferences randomly (in fact, $B_{2}$ is such a relation). Similarly, the behavioral preference relation $B_{1}$, which also obeys $E_{1}$ but is vulnerable to money pumps, resolves all indifferences randomly. By Proposition 9, amending it by resolving some or all indifferences selectively cannot make it invulnerable to money pumps.

\section{Money pumps and basic evaluation}

In the illustrative example, if two models $(s, q)$ and $(t, r)$ are offered at the same price $p$, then the first offer is judged at least as valuable as the second one if and only if either $s=t$ and $q=r$, or $s>t$ and $q>r$. This judgment, in fact, is independent of the price: it would not be altered if we replaced $p$ by any other price, as long as the price is common to the two models. In this sense, the evaluation of offers provided by the computing and accounting departments contains an underlying evaluation of computers alone. Such evaluations of basic objects alone are the topic of this section. An interesting feature of the results to come is that they do not rely on the assumptions that the company's evaluative preference relation $E$ is monotonic and Archimedean and that its behavioral preference relation $B$ is Archimedean, so from now on we merely assume that $E$ is reflexive and $B$ is complete.

\subsection{Basis of an evaluation}

Just as an evaluation of offers, an evaluation of computers can be modeled by an evaluative preference relation, only on the set $B O$ of computers rather than the set $X=B O \times M P$ of offers. Let us denote such a preference relation by $\hat{E}$, i.e. we write $b \hat{E} c$ if model $b$ is judged at least as valuable as model $c$. All general properties (not the monetary ones) of preferences defined in Subsection 2.2 can naturally be applied to $\hat{E}$ and, in particular, it is natural to assume that $\hat{E}$ is reflexive. That the evaluative preference relation $E_{1}$ on $X$ of the illustrative example contains an underlying evaluation of computers alone means, formally, that there exists an evaluative preference relation $\hat{E}_{1}$ on $B O$ such that, for any models $b=(s, q)$ and $c=(t, r)$ and any monetary payoff $m,(b, m) E_{1}(c, m)$ if and only if $b \hat{E}_{1} c$. In fact, as noted above, $\hat{E}_{1}$ is defined by $(s, q) \hat{E}_{1}(t, r)$ if and only if either $s=t$ and $q=r$, or $s>t$ and $q>r$. One can check that $\hat{E}_{1}$ is reflexive and transitive but not complete, reflecting non-exhaustiveness of the evaluation of computers.

Looking further at the illustrative example, we can observe the following relationships between the evaluation of offers and the underlying evaluation of computers. First, if model $b$ is judged at least as valuable as model $c$ and is offered at a strictly lower price than model $c$, then the former offer is judged strictly more valuable than the latter. Second, if model $b$ is judged strictly more valuable than model $c$, then this judgment is monetarily substantial in the sense 
that even if model $b$ is offered at a slightly higher price than model $c$, the former offer remains strictly more valuable than the latter. Third, if models $b$ and $c$ are mutually unevaluated, then this lack of evaluation is also monetarily substantial in the sense that some slight difference between the two model's prices (in one direction or the other) leaves the two offers mutually unevaluated. We will say that an underlying evaluation of computers satisfying these three properties is a basis of the evaluation of options:

Definition. $\hat{E}$ is a basis of $E$ if, for any $b, c \in B O$ and any $m \in M P$ :

- $(b, m) E(c, m)$ if and only if $b \hat{E} c$,

- if $b \hat{E} c$ then $(b, m) P(E)(c, n)$ for any $n \in M P$ such that $m>n$,

- if $b P(\hat{E}) c$ then there exists some $n \in M P$ such that $m<n$ and $(b, m) P(E)(c, n)$,

- if $b N(\hat{E}) c$ then there exists some $n \in M P$ such that $m \neq n$ and $(b, m) N(E)(c, n)$.

Any evaluation of computers can serve as basis for some evaluation of offers (for instance, among any two offers, the company could systematically judge more valuable the one with the most valuable computer, if there is one, and the one with the lowest price otherwise). Moreover, an evaluation of offers obviously cannot have more than one basis. But it may also have no basis at all. Indeed, first, an evaluation of offers does not necessarily contain an underlying evaluation of computers (for instance, a company could judge model $b$ strictly more valuable than model $c$ if both models are offered at a low, common price but judge model $c$ strictly more valuable than model $b$ if both models are offered at a high, common price). Second, even if the evaluation of offers contains an underlying evaluation of computers, this underlying evaluation is not necessarily a basis (for instance, a company could use an evaluation of computers only to choose between offers that have a common price and systematically select the offer with the lowest price otherwise, in which case underlying strict preference is not monetarily substantial).

\subsection{Basic invulnerability to money pumps}

If the company's evaluation of offers is not potentially invulnerable to money pumps, and if the company wishes to modify it so as tho make it potentially invulnerable to money pumps, then they might want to know, roughly speaking, how deeply they need to reevaluate offers. If the evaluation, in addition, has a basis, then we can give an element of answer by asking the following, simpler question: is it possible to make the evaluation of offers potentially invulnerable to money pumps while retaining its basis? Equivalently, we want to determine when can an evaluative preference relation $\hat{E}$ over basic objects serve as basis for some evaluative preference relation $E$ over offers that is potentially invulnerable to money pumps:

Definition. $\hat{E}$ is basically invulnerable to money pumps if there exists some reflexive $E$ such that $\hat{E}$ is the basis of $E$ and some complete $B$ that is invulnerable to money pumps and obeys $E$.

The following proposition gives a necessary and sufficient condition for $\hat{E}$ to be basically invulnerable to money pumps: 
Proposition 10. Assume that $\hat{E}$ is reflexive. Then $\hat{E}$ is basically invulnerable to money pumps if and only if $\hat{E}$ is consistent.

As with potential invulnerability to money pumps, consistency is sufficient to guarantee basic invulnerability to money pumps, but this time it is also necessary. Evaluations of offers that have a consistent basis can be made potentially invulnerable to money pumps (if they are not already) while retaining the basis, whereas evaluations of offers that have an inconsistent basis cannot. In the illustrative example, the evaluative preference relation $\hat{E}_{1}$ over computers is transitive and, hence, consistent, so it can serve as basis for an evaluation of offers that is potentially invulnerable to money pumps (in fact, the evaluative preference relation $E_{1}$ corresponds to such an evaluation of offers). Of course, this says nothing about evaluations of offers that have no basis at all (and that may also be potentially invulnerable to money pumps or not). Also, as with evaluation of offers, non-exhaustiveness of the basic evaluation of computers does not preclude invulnerability to money pumps. In order to investigate whether such non-exhaustiveness calls for specific choice behavior in order to avoid money pumps, we now investigate how resolution of noncomparability and indifference affects basic invulnerability to money pumps:

Definition. $\hat{E}$ is basically invulnerable to money pumps without selective resolution of noncomparability (resp. indifference) if there exists some reflexive $E$ such that $\hat{E}$ is the basis of $E$ and some complete $B$ that is invulnerable to money pumps, obeys $E$ and resolves all noncomparabilities (resp. indifferences) randomly.

Concerning noncomparability, we now obtain a necessary and sufficient condition:

Proposition 11. Assume that $\hat{E}$ is reflexive. Then $\hat{E}$ is basically invulnerable to money pumps without selective resolution of noncomparability if and only if $\hat{E}$ is complete and transitive.

In the illustrative example, the evaluative preference relation $\hat{E}_{1}$ over computers is transitive but not complete, so it cannot serve as basis for an evaluation of offers that is potentially invulnerable without selective resolution of noncomparability. That is to say, not only is the evaluative preference $E_{1}$ over offers not invulnerable to money pumps without selective resolution of noncomparability, but it is also impossible to modify $E_{1}$ so as to make it invulnerable to money pumps without selective resolution of noncomparability while retaining $\hat{E}_{1}$ as a basis. Thus if $E_{1}$ is to serve as basis and money pumps are to be avoided, then selective resolution of at least some noncomparabilities is necessary. Comparing Propositions 10 and 11, it appears that whenever the evaluation of options is coherent but non-exhaustive, it can serve as basis of an evaluation of offers without exposing the company to money pumps, but only if at least some noncomparabilities between offers are resolved selectively:

Proposition 12. Assume that $\hat{E}$ is reflexive. If $\hat{E}$ is consistent but not complete, then $\hat{E}$ is basically invulnerable to money pumps but not without selective resolution of noncomparability.

Concerning indifference, we obtain the same result as with evaluation of offers:

Proposition 13. Assume that $\hat{E}$ is reflexive. If $\hat{E}$ is basically invulnerable to money pumps, then $\hat{E}$ is basically invulnerable to money pumps without selective resolution of indifference. 
Finally, these results on basic evaluations can easily be linked to the original money pump argument by Davidson, McKinsey, and Suppes (1955). Although their argument is informal, it can be formally stated as follows within our framework:

Proposition (Davidson, McKinsey, and Suppes, 1955). Assume that $\hat{E}$ is complete. Then $\hat{E}$ is basically invulnerable to money pumps without selective resolution of indifference if and only if $\hat{E}$ is transitive.

Since consistency and transitivity of $\hat{E}$ are equivalent when $\hat{E}$ is complete, this is clearly a consequence of Propositions 10 and 13. Proposition 13 in fact shows that the requirement that all indifferences be resolved randomly could equivalently be dispensed with. Moreover, if the assumption that $\hat{E}$ is complete is dropped and replaced with the requirement that all noncomparabilities be resolved randomly then, by Proposition 11, basic invulnerability to money pumps is characterized by completeness and transitivity together. This can be seen as giving foundations to the completeness assumption in Davidson, McKinsey, and Suppes (1955)'s argument. However, if random resolution of all noncomparabilities is not a priori imposed then, by Proposition 10, completeness is no longer necessary for basic invulnerability to money pumps and, consequently, transitivity is generalized to consistency.

\section{Conclusion}

How should a decision-maker, such as a company, choose in the absence of evaluative preference? That is to say, should a company facing alternative options that they either have not mutually evaluated (noncomparability) or have judged equally valuable (indifference), choose randomly (in some way or another) between these options or rather deliberately select one of them, even though there is no evaluation on which to base such a selection? Although random choice may appear as the simplest solution in practice, we saw that deliberate selection may potentially reduce the company's exposition to a specific type of monetary loss, namely, money pumps. We undertook to determine if and when it actually does so.

Our first task was to determine when are money pumps avoidable at all. We found that the company can protect themselves against money pumps if and only if their evaluation of options gives rise to a (complete or incomplete) ranking of options satisfying a consistency property that expresses a weak form of coherence of the evaluation (consistency is equivalent to the well-known transitivity property if the ranking is complete and weaker than transitivity if the ranking is incomplete). If the ranking is inconsistent, then the company is exposed to money pumps no matter how they choose in the absence of preference. If the ranking is consistent then two situations may arise, depending on whether the ranking is complete or incomplete. If the ranking is complete then the company is protected from money pumps no matter how they choose in the absence of preference. If the ranking is incomplete then the company may or may not be exposed to money pumps, depending on how they choose between noncomparable or indifferent options. More precisely, the company can always protect themselves from money pumps by deliberately selecting among noncomparable options in an appropriate, consistent way, but will always be exposed to money pumps if they systematically choose randomly between noncomparable options. Whether the company chooses randomly or make a deliberate selection 


\begin{tabular}{|c|c|c|}
\hline $\begin{array}{c}\text { When evaluation } \\
\text { of options is... }\end{array}$ & complete & incomplete \\
\hline consistent & No & $\begin{array}{c}\text { No if selection } \\
\text { Yes if randomization }\end{array}$ \\
\hline inconsistent & Yes & Yes \\
\hline
\end{tabular}

Figure 2: Is a decision-maker exposed to money pumps?

among indifferent options, on the other hand, can neither protect them against nor expose them to money pumps. These findings are summarized in Figure 2.

The consistency of evaluation that is necessary for money pumps to be avoidable is a global property, in the sense that it involves all options at once. That is to say, splitting the set of available options into two subsets and checking for consistency of the evaluation on each subset separately by no means guarantees consistency of the evaluation on the whole set. Thus, in order to avoid money pumps, the company's evaluation process should be assessed at a global rather than local level (taking the decision-maker to be a social planner and using a related, Dutch book argument, Diecidue, 2006 reaches a similar conclusion concerning policy evaluation). Furthermore, if this process does not lead to a complete ranking of all available options (which may be natural in case of a high number of options, of the complexity of some options, of a lack of information, time constraints...) then, in order to avoid money pumps, the company will have to devise a consistent strategy for selecting among noncomparable options, and this strategy should also be assessed globally. In this sense, the present analysis highlights the potential benefit for managers of a global rather than local assessment of their organization's decision process.

\section{Appendix}

Proof of Proposition 1. Assume that $B$ is monotonic and $M(B)$ is transitive. Suppose that there exist some $b \in B O$ and $m, n \in M P$ such that $n<m$ and $(b, n) T(B)(b, m)$. Then $(b, n) T(M(B))(b, m)$ because $B$ is monotonic and, hence, $(b, n) M(B)(b, m)$ because $M(B)$ is transitive. Hence $(b, m) P(B)(b, m)$ because $m>n$, a contradiction. Hence $B$ is invulnerable to money pumps.

Conversely, first, if $B$ is not monotonic then there exist $(b, m),(c, n) \in X$ and $o \in M P$ such that $o>m,(b, m) B(c, n)$, and not $(b, o) P(B)(c, n)$. Hence $(c, n) B(b, o)$ since $B$ is complete and, hence, $(b, m) T(B)(b, o)$, so $B$ is not invulnerable to money pumps since $m<o$. Second, assume that $M(B)$ is intransitive, i.e. there exist some $(b, m),(c, n) \in X$ such that $(b, m) T(M(B))(c, n)$ and not $(b, m) M(B)(c, n)$. Then there exist some integer $k \geq 1$ and some sequence $\left(b_{0}, m_{0}\right),\left(b_{1}, m_{1}\right), \ldots,\left(b_{k}, m_{k}\right) \in X$ such that $\left(b_{0}, m_{0}\right) M(B)\left(b_{1}, m_{1}\right)$, $\ldots,\left(b_{k-1}, m_{k-1}\right) M(B)\left(b_{k}, m_{k}\right),\left(b_{0}, m_{0}\right)=(b, m)$, and $\left(b_{k}, m_{k}\right)=(c, n)$. It follows that not $\left(b_{0}, m_{0}\right) M(B)\left(b_{k}, m_{k}\right)$ by definition and, hence, there exists some $n_{0} \in M P$ such that $n_{0}>m_{0}$ and $\left(b_{k}, m_{k}\right) B\left(b_{0}, n_{0}\right)$ since $B$ is complete. Let $o_{0} \in M P$ such that $n_{0}>o_{0}>$ $m_{0}$. Then $\left(b_{0}, o_{0}\right) P(B)\left(b_{1}, m_{1}\right)$ by definition and, hence, there exists some $n_{1} \in M P$ such that $n_{1}>m_{1}$ and $\left(b_{0}, n_{0}\right) B\left(b_{1}, n_{1}\right)$ since $B$ is Archimedean. By induction, there exists 
some sequence $n_{1}, \ldots, n_{k} \in M P$ such that $n_{1}>m_{1}, \ldots, n_{k}>m_{k},\left(b_{0}, n_{0}\right) B\left(b_{1}, n_{1}\right), \ldots$, $\left(b_{k-1}, n_{k-1}\right) B\left(b_{k}, n_{k}\right)$. Hence $\left(b_{k}, m_{k}\right) T(B)\left(b_{k}, n_{k}\right)$, so $B$ is not invulnerable to money pumps since $m_{k}<n_{k}$.

Proof of Proposition 2. Assume that $B$ is transitive. Suppose that there exist some $b \in B O$ and $m, n \in M P$ such that $n<m$ and $(b, n) T(B)(b, m)$. Then $(b, n) B(b, m)$ because $B$ and, hence, $(b, n) M(B)(b, m)$ because $B$ is transitive and monotonic. Hence $(b, m) P(B)(b, m)$ because $m>n$, a contradiction. Hence $B$ is invulnerable to money pumps.

Lemma 1. Let $R$ be an Archimedean preference relation on $X$. Then (i) $M(R)$ is monotonic and Archimedean, and (ii) $M(M(R))=M(R)$.

Proof. (i). Let $(b, m),(c, n) \in X$ such that $(b, m) M(R)(c, n)$, and let $o \in M P$ such that $o>m$. Then $(b, o) M(R)(c, n)$ by definition. Suppose that $(c, n) M(R)(b, o)$, and let $p \in M P$ such that $o>p>m$. Then $(b, p) P(R)(c, n)$ and, hence, there exists some $q \in M P$ such that $q>n$ and $(b, o) R(c, q)$ by Archimedeanness of $R$, a contradiction because $(c, n) M(R)(b, o)$. Hence $(b, o) P(M(R))(c, n)$, so $M(R)$ is monotonic. Hence $(b, o) R(c, q)$ implies $(b, o) M(R)(c, q)$ by monotonicity of $R$, so $M(R)$ is Archimedean. (ii). Let $(b, m),(c, n) \in X$. If $(b, m) M(R)(c, n)$, then $(b, m) M(M(R))(c, n)$ because $M(R)$ is monotonic. Conversely, assume that $(b, m) M(M(R))(c, n)$ and let $o \in M P$ such that $o>m$. Let $p \in M P$ such that $o>p>m$. Then $(b, p) P(M(R))(c, n)$ and, hence, $(b, o) P(R)(c, n)$ by definition, so $(b, m) M(R)(c, n)$.

Proof of Proposition 3. First note that $M(B)$ is complete because $B$ is complete and monotonic. Also, $M(B)$ is monotonic and Archimedean by Lemma 1 because $B$ is Archimedean. By Proposition 1, $B$ is invulnerable to money pumps if and only if $M(B)$ is transitive, and $M(B)$ is invulnerable to money pumps if and only if $M(M(B))$ is transitive, i.e. if and only if $B$ is invulnerable to money pumps since $M(M(B))=M(B)$ by Lemma 1 .

Lemma 2. Assume that there exists some complete and Archimedean $B$ that is invulnerable to money pumps and such that, for any $(b, m),(c, n) \in X$, if $(b, m) E(c, n)$ then $(b, m) B(c, n)$. Then $E$ is potentially invulnerable to money pumps without selective resolution of indifference.

Proof. Define the preference relation $B^{*}$ on $X$ by $(b, m) B^{*}(c, n)$ if and only if $(b, m) B(c, n)$ and not $(c, n) P(E)(b, m)$. Then $B^{*}$ is complete, obeys $E$, and resolves all indifferences randomly by definition. Moreover, suppose that there exist some $b \in B O$ and $m, n \in M P$ such that $n<m$ and $(b, n) T\left(B^{*}\right)(b, m)$. Then $(b, n) T(B)(b, m)$ by definition of $B^{*}$, a contradiction because $B$ is invulnerable to money pumps. Hence $B^{*}$ is invulnerable to money pumps. It only remains to prove that $B^{*}$ is monotonic and Archimedean. To this end, first note that $B$ is monotonic by Proposition 1. Now, let $(b, m),(c, n) \in X$ such that $(b, m) B^{*}(c, n)$, and let $o \in M P$ such that $o>m$. Then $(b, m) B(c, n)$ by definition and, hence, $(b, o) P(B)(c, n)$ since $B$ is monotonic. Hence not $(c, n) B^{*}(b, o)$ by definition and, hence, $(b, o) P\left(B^{*}\right)(c, n)$ since $B^{*}$ is complete, so $B^{*}$ is monotonic. Moreover, let $p \in M P$ such that $o>p>m$. Since $B$ is Archimedean, there exists some $q \in M P$ such that $q>n$ and $(b, p) B(c, q)$ and, hence, $(b, o) P\left(B^{*}\right)(c, q)$ by the same argument as above, so $B^{*}$ is Archimedean. 
Proof of Propositions 6 and 9. First note that $M(E)$ is reflexive because $E$ is reflexive and monotonic. Also, $M(E)$ is monotonic and Archimedean by Lemma 1 because $E$ is Archimedean. Hence it is sufficient to prove that the three following statements are equivalent: (i) $E$ is potentially invulnerable to money pumps, (ii) $M(E)$ is potentially invulnerable to money pumps without selective resolution of indifference, and (iii) $E$ is potentially invulnerable to money pumps without selective resolution of indifference. Moreover, (iii) obviously implies (i), so it only remains to prove that (i) implies (ii) and (ii) implies (iii).

(i) implies (ii). Assume that there exists some complete and Archimedean $B$ that is invulnerable to money pumps and obeys $E$. Then $M(B)$ is monotonic and Archimedean by Lemma 1 because $B$ is Archimedean. Moreover, $B$ is monotonic by Proposition 1 and, hence, $M(B)$ is complete and invulnerable to money pumps by Proposition 3. Finally, for any $(b, m),(c, n) \in X$, if $(b, m) M(E)(c, n)$ then $(b, m) M(B)(c, n)$ because $B$ obeys $E$. Hence $M(E)$ is potentially invulnerable to money pumps without selective resolution of indifference by Lemma 2 .

(ii) implies (iii). Assume that there exists some complete and Archimedean $B$ that is invulnerable to money pumps, obeys $M(E)$, and resolves all indifferences randomly. Then, for any $(b, m),(c, n) \in X$, if $(b, m) E(c, n)$ then $(b, m) M(E)(c, n)$ because $E$ is monotonic and, hence, $(b, m) B(c, n)$ because $B$ obeys $M(E)$ and resolves all indifferences randomly. Hence $E$ is potentially invulnerable to money pumps without selective resolution of indifference by Lemma 2.

Lemma 3. Let $R$ be a preference relation on $X$. Then (i) if $R$ is Archimedean then $T(R)$ is Archimedean, and (ii) if $R$ is monotonic and consistent then $T(R)$ is monotonic.

Proof. To prove both statements, let $(b, m),(c, n) \in X$ such that $(b, m) T(R)(c, n)$, i.e. there exist some integer $k \geq 1$ and some sequence $\left(b_{0}, m_{0}\right),\left(b_{1}, m_{1}\right), \ldots,\left(b_{k}, m_{k}\right) \in X$ such that $\left(b_{0}, m_{0}\right) R\left(b_{1}, m_{1}\right), \ldots,\left(b_{k-1}, m_{k-1}\right) R\left(b_{k}, m_{k}\right),\left(b_{0}, m_{0}\right)=(b, m)$, and $\left(b_{k}, m_{k}\right)=(c, n)$. Let $n_{0} \in M P$ such that $n_{0}>m_{0}$. (i). Assume that $R$ is Archimedean. Then, by induction, there exists some sequence $n_{1}, \ldots, n_{k} \in M P$ such that $n_{1}>m_{1}, \ldots, n_{k}>m_{k},\left(b_{0}, n_{0}\right) R\left(b_{1}, n_{1}\right), \ldots$, $\left(b_{k-1}, n_{k-1}\right) R\left(b_{k}, n_{k}\right)$. Hence $\left(b_{0}, n_{0}\right) T(R)\left(b_{k}, n_{k}\right)$, so $T(R)$ is Archimedean. (ii). Assume that $R$ is monotonic and consistent. Then $\left(b_{0}, n_{0}\right) P(R)\left(b_{1}, m_{1}\right)$ and, hence, $\left(b_{0}, n_{0}\right) T(R)\left(b_{k}, m_{k}\right)$. Moreover, suppose $\left(b_{k}, m_{k}\right) T(R)\left(b_{0}, n_{0}\right)$. Then $\left(b_{1}, m_{1}\right) T(R)\left(b_{0}, n_{0}\right)$, a contradiction since $\left(b_{0}, n_{0}\right) P(R)\left(b_{1}, n_{1}\right)$ and $R$ is consistent. Hence $\left(b_{0}, n_{0}\right) P(T(R))\left(b_{k}, m_{k}\right)$, so $T(R)$ is monotonic.

Lemma 4. Let $R$ be a reflexive, monotonic, and transitive preference relation on $X$. Let $G, H \subseteq$ $X$ such that $(c, n) R(b, m)$ for no $(b, m) \in G$ and $(c, n) \in H$. Define the preference relation $R^{*}$ on $X$ by $(b, m) R^{*}(c, n)$ if and only if either $(b, m) R(c, n)$, or $(b, m) \in G$ and $(c, n) \in H$. Then $T\left(R^{*}\right)$ is reflexive, monotonic, obeys $R$, and resolves all indifferences randomly.

Proof. Let $(b, m),(c, n) \in X$. If $(b, m) R(c, n)$, then, by definition, $(b, m) R^{*}(c, n)$ and, hence, $(b, m) T\left(R^{*}\right)(c, n)$. Hence $T\left(R^{*}\right)$ is reflexive because $R$ is reflexive. Moreover, $(b, m) I(R)(c, n)$ implies $(b, m) I\left(T\left(R^{*}\right)\right)(c, m)$, so $T\left(R^{*}\right)$ resolves all indifferences randomly.

Now, assume that $(b, m) P(R)(c, n)$. By definition $(b, m) T\left(R^{*}\right)(c, n)$. Suppose that not $(b, m) P\left(T\left(R^{*}\right)\right)(c, n)$. Then $(c, n) T\left(R^{*}\right)(b, m)$ and, hence, there exist some integer $k \geq 1$ 
and some sequence $\left(b_{0}, m_{0}\right),\left(b_{1}, m_{1}\right), \ldots,\left(b_{k}, m_{k}\right) \in X$ such that $\left(b_{0}, m_{0}\right) R^{*}\left(b_{1}, m_{1}\right), \ldots$, $\left(b_{k-1}, m_{k-1}\right) R^{*}\left(b_{k}, m_{k}\right),\left(b_{0}, m_{0}\right)=(c, n)$, and $\left(b_{k}, m_{k}\right)=(b, m)$. Hence, there exists some integer $h$ such that $1 \leq h \leq k,\left(b_{h-1}, m_{h-1}\right) \in G$, and $\left(b_{h}, m_{h}\right) \in H$ (otherwise we would have $(c, n) R(b, m)$ by transitivity of $R$, a contradiction). One can, consequently, define the smallest integer $i$ such that $0 \leq i \leq k$ and $\left(b_{i}, m_{i}\right) \in G$, as well as the largest integer $j$ such that $0 \leq j \leq k$ and $\left(b_{j}, m_{j}\right) \in H$. Then $\left(b_{j}, m_{j}\right) T(R)(b, m)$ and $(c, n) T(R)\left(b_{i}, m_{i}\right)$ by definition of $R^{*}$. Hence $\left(b_{j}, m_{j}\right) P(R)\left(b_{i}, m_{i}\right)$ because $(b, m) P(R)(c, n)$ and $R$ is transitive, a contradiction because $\left(b_{i}, m_{i}\right) \in G$ and $\left(b_{j}, m_{j}\right) \in H$. Hence $(b, m) P\left(T\left(R^{*}\right)\right)(c, n)$, so $T\left(R^{*}\right)$ obeys $R$.

Finally, assume that $(b, m) T\left(R^{*}\right)(c, n)$, and let $o \in M P$ such that $o>m$. Then $(b, o) P(R)(b, m)$ because $R$ is reflexive and monotonic. Hence $(b, o) P\left(T\left(R^{*}\right)\right)(b, m)$ because $T\left(R^{*}\right)$ obeys $R$. Hence $(b, o) P\left(T\left(R^{*}\right)\right)(c, n)$ because $T\left(R^{*}\right)$ is transitive by definition, so $T\left(R^{*}\right)$ is monotonic.

Proof of Proposition 5. Assume that $E$ is consistent. By Proposition 2, it is sufficient to prove that there exists some complete, monotonic, Archimedean, and transitive $B$ that obeys $E$. In fact, we show that there exists one that also resolves all indifferences randomly. The proof is similar to that of Duggan (1999)'s General Extension Theorem.

Denote by $V$ the set of all reflexive, monotonic, Archimedean, and transitive preference relations on $X$ that obey $E$ and resolve all indifferences randomly. By Lemma 3 and Duggan (1999)'s Propositions 3 and $6, T(E) \in V$, so $V \neq \emptyset$. Let $W$ be a subset of $V$ such that, for any $R, R^{\prime} \in W$, either $R \subseteq R^{\prime}$ or $R^{\prime} \subseteq R$. Define the preference relation $R^{*}$ on $X$ by, for any $(b, m),(c, n) \in X,(b, m) R^{*}(c, n)$ if and only if there exists some $R \in W$ such that $(b, m) R(c, n)$. Let us show that $R^{*} \in V$. By Duggan (1999)'s Proposition 5, it is sufficient to show that $R^{*}$ is monotonic and Archimedean. Let $(b, m),(c, n) \in X$ such that $(b, m) R^{*}(c, n)$, and let $o \in M P$ such that $o>m$. By definition of $R^{*}$, there exists some $R \in W$ such that $(b, m) R(c, n)$. Hence, by Archimedeanness of $R$, there exists some $p \in M P$ such that $p>n$ and $(b, o) R(c, p)$ and, hence, $(b, o) R^{*}(c, p)$ by definition of $R^{*}$, so $R^{*}$ is Archimedean. Moreover, $(b, o) P(R)(c, n)$ because $R$ is monotonic, so $(b, o) R^{*}(c, n)$ by definition of $R^{*}$. Suppose that $(c, n) R^{*}(b, o)$. Then there exists some $R^{\prime} \in W$ such that $(c, n) R^{\prime}(b, o)$ by definition of $R^{*}$ and, hence, not $R^{\prime} \subseteq R$ because not $(c, n) R(b, o)$. Hence $R \subseteq R^{\prime}$ by definition of $W$ and, hence, $(b, m) R^{\prime}(c, n)$, a contradiction because $R^{\prime}$ is monotonic and $o>m$. Hence $(b, o) P\left(R^{*}\right)(c, n)$, so $R^{*}$ is monotonic.

By Zorn's lemma, there then exists some $B \in V$ such that, for any $R \in V, B \subseteq R$ implies $B=R$. It only remains to prove that $B$ is complete. Suppose that $B$ is incomplete. Then there exist some $(b, m),(c, n) \in X$ such that $(b, m) N(B)(c, n)$. In order to establish a contradiction, it is sufficient, by Duggan (1999)'s Proposition 3, to find some reflexive, monotonic, Archimedean, and transitive preference relation $B^{\prime}$ on $X$ that obeys $B$, resolves all indifferences randomly, and is such that either $(b, m) B^{\prime}(c, n)$ or $(c, n) B^{\prime}(b, m)$. Let us distinguish two cases.

Case 1. Assume that, for any $o \in M P$ such that $o>m$, there exists some $p \in M P$ such that $p>n$ and $(b, o) B(c, p)$. Let $G=\{(b, m)\}$ and $H=\{(c, n)\}$. Then, clearly, $(c, n) B(b, m)$ for no $(b, m) \in G$ and $(c, n) \in H$. Define the preference relation $B^{*}$ on $X$ as in Lemma 4 , and let $B^{\prime}=T\left(B^{*}\right)$. Then $B^{*}$ is Archimedean because $B$ is Archimedean and, hence, by Lemmas 3 and $4, B^{\prime}$ is reflexive, monotonic, Archimedean, obeys $B$, and resolves all indifferences randomly. 
Moreover, $B^{\prime}$ is transitive and $(b, m) B^{\prime}(c, n)$ by definition, so the case is complete.

Case 2. If Case 1 does not apply, then there exists some $o \in M P$ such that $o>m$ and, for any $p \in M P$ such that $p>n$, not $(b, o) B(c, p)$. Let $G=\{(c, p): p \in M P, p \geq n\}$ and $H=\{(b, q): q \in M P, o>q \geq m\}$. Then $(b, q) B(c, p)$ for no $(c, p) \in G$ and $(b, q) \in$ $H$ (otherwise there would exist some $r \in M P$ such that $r>p>n$ and $(b, o) B(c, r)$ by Archimedeanness of $B$, a contradiction). Define the preference relation $B^{*}$ on $X$ as in Lemma 4 , and let $B^{\prime}=T\left(B^{*}\right)$. Then $B^{*}$ is Archimedean because $B$ is Archimedean and, hence, by Lemmas 3 and $4, B^{\prime}$ is reflexive, monotonic, Archimedean, obeys $B$, and resolves all indifferences randomly. Moreover, $B^{\prime}$ is transitive and $(c, n) B^{\prime}(b, m)$ by definition, so the case is complete and the desired contradiction is established, proving that $B$ is complete.

Proof of Proposition 4. First note that $M(E)$ is reflexive because $E$ is reflexive and monotonic. Also, $M(E)$ is monotonic and Archimedean by Lemma 1 since $E$ is Archimedean. Assume that $M(E)$ is consistent. Then $M(E)$ is potentially invulnerable to money pumps by Proposition 5. Hence $E$ is potentially invulnerable to money pumps by Proposition 6 .

Proof of Proposition 7. Assume that there exists some complete and Archimedean $B$ that is invulnerable to money pumps, obeys $E$, and resolves all noncomparabilities randomly. Suppose that $M(E)$ is incomplete, i.e. there exist some $(b, m),(c, n) \in X$ such that $(b, m) N(M(E))(c, n)$. Then there exist some $o, p \in M P$ such that $o>m, p>n$, not $(b, o) P(E)(c, n)$, and not $(c, p) P(E)(b, m)$ by definition of $M(E)$. Let $q, r \in M P$ such that $o>q>m$ and $p>r>n$. Then not $(b, q) E(c, n)$ and not $(c, r) E(b, m)$ since $E$ is monotonic. Hence $(c, n) B(b, q)$ and $(b, m) B(c, r)$ since $B$ obeys $E$ and resolve all noncomparabilities randomly. Moreover, we have $(c, r) P(E)(c, n)$ since $E$ is reflexive and monotonic and, hence, $(c, r) P(B)(c, n)$ since $B$ obeys $E$. Hence $(b, m) T(B)(b, q)$, so $B$ is not invulnerable to money pumps, a contradiction. Hence $M(E)$ is complete.

Now, since $E$ is potentially invulnerable to money pumps, so is $M(E)$ by Proposition 6 . Hence, by Proposition 9, there exists some complete and Archimedean $B^{\prime}$ that is invulnerable to money pumps, obeys $M(E)$, and resolves all indifferences randomly. By Proposition 1, it follows that $M\left(B^{\prime}\right)$ is transitive. Moreover, we have $B^{\prime}=M(E)$ since $M(E)$ is complete and $B^{\prime}$ resolves all indifferences randomly and, hence, $M\left(B^{\prime}\right)=M(M(E))=M(E)$ by Lemma 1 , so $M(E)$ is transitive.

Proof of Proposition 8. Follows directly from Propositions 4 and 7.

Proof of Propositions 10 and 13. It is sufficient to prove that if $\hat{E}$ is consistent then $\hat{E}$ is basically invulnerable to money pumps without selective resolution of indifference, whereas if $\hat{E}$ is not consistent then $\hat{E}$ is not basically invulnerable to money pumps. So assume that $\hat{E}$ is consistent. Define the evaluative preference relation $E$ on $X$ by $(b, m) E(c, n)$ if and only if either $b P(\hat{E}) c$, or $b I(\hat{E}) c$ and $m \geq n$. Then $\hat{E}$ is the basis of $E$ and $E$ is reflexive, monotonic, Archimedean, and consistent. Hence $E$ is potentially invulnerable to money pumps without selective resolution of indifference by Propositions 4 and 9 . Conversely, assume that $\hat{E}$ is not consistent, i.e. there exist some integer $k \geq 1$ and some sequence $b_{0}, b_{1}, \ldots, b_{k} \in B O$ such that $b_{0} \hat{E} b_{1}, \ldots, b_{k-1} \hat{E} b_{k}$, and $b_{k} P(\hat{E}) b_{0}$. Let $E$ be a reflexive preference relation 
on $X$ such that $\hat{E}$ is the basis of $E$, and let $m \in M$. Then by definition of a basis, there exists some $n \in M P$ such that $m<n$ and $\left(b_{k}, m\right) P(E)\left(b_{0}, n\right)$. Let $m_{0}, m_{1}, \ldots, m_{k} \in M P$ such that $n>m_{0}>m_{1}>\ldots>m_{k}=m$. Then, again by definition of a basis, we have $\left(b_{0}, m_{0}\right) P(E)\left(b_{1}, m_{1}\right), \ldots,\left(b_{k-1}, m_{k-1}\right) P(E)\left(b_{k}, m_{k}\right)$ and, hence, $\left(b_{0}, m_{0}\right) T(P(E))\left(b_{0}, n\right)$. Hence for any complete $B$ on $X$ that obeys $E$, we have $\left(b_{0}, m_{0}\right) T(P(B))\left(b_{0}, n\right)$, so $B$ is not invulnerable to money pumps since $m_{0}<n$.

Proof of Proposition 11. Recall that transitivity is equivalent to consistency for a complete preference relation. Hence, by Proposition 10, it is sufficient to show that if $\hat{E}$ is consistent, then $\hat{E}$ is invulnerable to money pumps without noncomparable selection if and only if $\hat{E}$ is complete. So assume that $\hat{E}$ is consistent. If $\hat{E}$ is complete, then the evaluative preference relation $E$ on $X$ defined in the proof of Propositions 10 and 13 is complete and, hence, potentially invulnerable to money pumps without selective resolution of noncomparability. Conversely, if $\hat{E}$ is not complete, i.e. there exist some $b, c \in B O$ such that $b N(\hat{E}) c$, then for any reflexive $E$ such that $\hat{E}$ is the basis of $E$ and any $m \in M P$, there exists some $n \in M P$ such that $m \neq n,(b, m) N(E)(c, m)$, and $(b, m) N(E)(c, n)$. Hence for any complete $B$ that obeys $E$ and resolves all noncomparabilities randomly, we have $(c, m) T(I(B))(c, n)$, so $B$ is not invulnerable to money pumps.

Proof of Proposition 12. Follows directly from Propositions 10 and 11.

\section{References}

Ariely, D., G. Loewenstein, and D. Prelec (2003): "Coherent arbitrariness": Stable demand curves without stable preferences," Quarterly Journal of Economics, 118(1), 73-105.

(2006): "Tom Sawyer and the construction of value," Journal of Economic Behavior and Organization, 60, 1-10.

Aumann, R. J. (1962): "Utility theory without the completeness axiom," Econometrica, 30(3), $445-462$.

Baucells, M., And L. S. Shapley (2008): "Multiperson utility," Games and Economic Behavior, 62, 329-347.

Bewley, T. F. (1986): "Knightian decision theory: Part I," Discussion Paper 807, Cowles Foundation Discussion Papers, published in Decisions in Economics and Finance (2002), 25, 79-110.

Bossert, W., Y. Sprumont, and K. Suzumura (2005): "Consistent Rationalizability," Economica, 72, 185-200.

Burros, R. H. (1974): "Axiomatic analysis of non-transitivity of preference and of indifference," Theory and Decision, 5, 185-204.

Chambers, R. G. (2009): "Uncertain equilibria and incomplete preferences," . 
Cubitt, R. P., And R. Sugden (2001): "On money pumps," Games and Economic Behavior, $37(1), 121-160$.

DANAN, E. (2003a): "A behavioral model of individual welfare," .

— (2003b): "Revealed cognitive preference theory," .

- (2008): "Revealed preference and indifferent selection," Mathematical Social Sciences, $55,24-37$.

Davidson, D., J. C. C. McKinsey, and P. Suppes (1955): "Outlines of a formal theory of value, I," Philosophy of Science, 22(2), 140-160.

Diecidue, E. (2006): "Deriving Harsanyi's utilitarianism from de Finetti's book-making argument," Theory and Decision, 61, 363-371.

Dubra, J., F. Maccheroni, And E. A. OK (2004): "Expected utility theory without the completeness axiom," Journal of Economic Theory, 115(1), 118-133.

Dubra, J., AND E. OK (2002): "A model of procedural decision making in the presence of risk," International Economic Review, 43(4), 1053-1080.

DugGan, J. (1999): "A general extension theorem for binary relations," Journal of Economic Theory, 86(1), 1-16.

Eliaz, K., And E. A. OK (2006): "Indifference or indecisiveness? Choice-theoretic foundations of incomplete preferences," Games and Economix Behavior, 56(1), 61-86.

Evren, O. (2008): "Continuous representation of (indecisiveness-driven) preference for flexibility," .

Evren, O., AND E. OK (2008): "On the multi-utility representation of incomplete preference relations," .

Gilboa, I., F. Maccheroni, M. Marinacci, and D. Schmeidler (2008): "Objective and subjective rationality in a multile prior model," .

Gursel TAPKI, I. (2007): "Revealed incomplete preferences under status-quo bias," Mathematical Social Sciences, 53(3), 274-283.

Kochov, A. S. (2007): "Subjective states without the completeness axiom," .

Lopomo, G., L. Rigotti, and C. Shannon (2008): "Uncertainty in mechanism design," .

Maccheroni, F. (2004): "Yaari's dual theory without the completeness axiom," Economic Theory, 23, 701-714.

MAndler, M. (2005): "Incomplete preferences and rational intransitivity of choice," Games and Economic Behavior, 50(2), 255-277. 
(2009): "Indifference and incompleteness distinguished by rational trade," Games and Economic Behavior, 67, 300-314.

Manzini, P., And M. Mariotti (2004): "How vague can one be? Rational preferences without completeness or transitivity," .

- (2008): "On the representation of incomplete preferences over risky alternatives," Theory and Decision, forthcoming.

Masatlioglu, Y., And E. A. OK (2005): "Rational choice with status quo bias," Journal of Economic Theory, 121(1), 1-29.

Nascimento, L. (2008): "On demand theory with incomplete preferences," .

Nascimento, L., And G. Riella (2008): "A class of incomplete and ambiguity averse preferences," .

Ok, E., P. Ortoleva, and G. Riella (2008): "Incomplete preferences under uncertainty: indecisiveness in beliefs vs. tastes," .

OK, E. A. (2002): "Utility representation of an incomplete preference relation," Journal of Economic Theory, 104(2), 429-449.

RIchter, M. K. (1971): "Rational choice," in Preferences, utility, and demand, ed. by J. S. Chipman, L. Hurwicz, M. K. Richter, and H. Sonnenschein. Harcourt Brace Jovanovich.

Rigotti, L., and C. Shannon (2005): "Uncertainty and risk in financial markets," Econometrica, 73(1), 203-243.

SEn, A. K. (1971): "Choice functions and revealed preference," Review of Economic Studies, $38(3), 307-317$.

Suzumura, K. (1976): "Remarks on the theory of collective choice," Economica, New Series, 43(172), 381-390. 